\title{
Efeitos de variáveis cognitivas, emocionais e atitudinais no rendimento a
}

\section{matemática}

\section{Effects of cognitive, emotional, and attitudinal variables on mathematics performance}

\author{
Adelinda Candeias, Inês Calisto, Ana Maria Cristóvão, José Verdasca, Paulo Vília \\ Universidade de Évora
}

\begin{abstract}
Resumo
Para explicar o rendimento a matemática e as suas causas têm sido apontadas diversas variáveis preditoras que deixam antever a complexidade e a combinação de múltiplas variáveis. Alguns estudos apontam a natureza multifatorial do desempenho a matemática, em que se combinam dimensões cognitivas e afetivas do indivíduo e os processos de ensino-aprendizagem da matemática. $\mathrm{O}$ objetivo principal deste estudo é avaliar o impacto das variáveis cognitivas, emocionais e atitudinais no desempenho a matemática. Os resultados destacam a necessidade de olhar o rendimento a matemática no $4^{\circ}$ ano de escolaridade através de uma abordagem multivariada. Palavras-chave: Rendimento escolar a matemática; $1^{\circ}$ ciclo do ensino básico; Variáveis cognitivas; Variáveis emocionais.
\end{abstract}

\begin{abstract}
Several predictive variables have been proposed to explain achievement on mathematics, allowing for the anticipation of complexity and the combination of multiple variables. Some studies indicate the multifactorial nature of mathematical performance, combining the cognitive and affective dimensions of the individual and the teaching and learning processes of mathematics. The main objective of this study is to evaluate the impact of cognitive, emotional and attitudinal variables on math performance. The results highlight the need to look at mathematics achievement in the 4th year of school using a multivariate approach.

Keywords: Mathematics school performance; 1st cycle of basic education; Cognitive variables; Emotional variables
\end{abstract}

\section{Introdução}

A matemática é uma ciência que lida com objetos e relações abstratas. Esta compreende uma linguagem que permite elaborar uma compreensão e representação do mundo que nos rodeia, ao mesmo tempo que nos proporciona formas de agir sobre este, aquando a necessidade de resolver problemas e de prever e controlar resultados das nossas próprias ações (Ponte et al., 2007). Atualmente, ser bom a matemática não é ser bom a fazer contas ou a "medir", mas sim ser alguém competente para fazer perguntas e seguir incansavelmente as pistas que emergem, procurando alcançar respostas que mostrem perspetivas divergentes sobre olhar a realidade (Leandro,
2006). Neste sentido, o ensino desempenha um papel fundamental para que todos os alunos tenham uma formação sólida a matemática (Gonzáles-Pienda et al., 2006 a, b; Ponte et al., 2007). Ou seja, uma formação que permita aos alunos compreender e utilizar a matemática, não apenas nas diferentes disciplinas em que é necessária, mas futuramente, a nível profissional e pessoal. Da mesma forma é importante que seja incutida aos alunos uma visão adequada da matemática e da sua atividade, assim como o reconhecimento dos seus contributos para o desenvolvimento científico e tecnológico da sociedade assim como a sua importância cultural e social. Em síntese, é importante promover-se junto dos alunos a aquisição da informação, do conhecimento e da experiência em matemática, assim como a capacidade para a integração e mobilização das competências adquiridas nos diferentes contextos de vida social e para o exercício da cidadania, gerando atitudes positivas face à matemática como uma ferramenta essencial para o desenvolvimento pessoal e social.

Efklides (2009) propõe uma abordagem à aprendizagem como um processo dinâmico e complexo, mas integrado, que envolve não apenas a cognição, mas também a motivação, o afeto, a volição e a metacognição. A metacognição é, brevemente, definida como o conhecimento que os alunos têm relativamente às suas operações mentais e, embora exista uma panóplia de definições encontramos, em comum, o conhecimento e a regulação das próprias aprendizagens (Rosário, 1997). Esta associação entre aprendizagem autorregulada e afeto torna-se evidente quando o sucesso ou o fracasso se manifestam no self do aluno e na sua auto-percepção de competência (Efklides, 2009). Porque o nosso comportamento social é fortemente influenciado pelos nossos sentimentos torna-se importante abordarmos o papel da afetividade na aprendizagem (Silva \& Candeias, 2012).

Os problemas da matemática têm uma origem intrapessoal e são iniciados a partir das caraterísticas pessoais nos processos mentais de aprendizagem dos alunos, das suas atitudes, aptidão cognitiva e motivação (Mohsenpour, Hejazi, Kiamanesh, 2008). 
Os estudos relacionados com o domínio afetivo e a educação matemática viram no estudo das atitudes face à matemática uma tentativa de descrever e analisar todos os componentes do domínio afetivo (McLeod, 1992). McLeod (1992) associa o domínio afetivo a um amplo espectro de crenças, sentimentos e atitudes, relacionados com o domínio cognitivo. Os três constructos variam na estabilidade das respostas afetivas que representam, enquanto as crenças e as atitudes são, geralmente, estáveis, as emoções podem variar rapidamente; no nível de intensidade dos afetos descrevem; e, no grau em que a cognição está presente na resposta e no tempo que leva a ser desenvolvida.

As atitudes são um conceito importante no campo da aprendizagem da matemática. Como é do nosso conhecimento muitos estudantes têm uma atitude negativa em relação à matemática que pode ser descrita como "ansiedade matemática", no entanto, essa dificuldade não está relacionada com a competência, mas sim com as atitudes que são realizadas sobre matemática (Yenilmez, Girginer, \& Uzun, 2007). Na opinião de Roesken, Hannula e Pehkonen (2011) a visão que os alunos têm sobre si mesmos como aprendizes de matemática é um critério decisivo para o seu empenho e sucesso na escola.

As investigações recentes demonstram um crescente reconhecimento do papel dos fatores afetivos no ensino e aprendizagem da matemática (Colomeischi e Colomeischi, 2016). A questão emocional relacionada com o desempenho escolar tem ganho importância nas últimas década, como é sabido as emoções podem facilitar ou impedir o empenho e sucesso escolar dos alunos, as relações e os processos emocionais afetam como e o que aprendemos, (Elias et al. 1997). Segundo Larkin e Jorgensen (2016) a investigação sobre crenças, atitudes e emoções indica uma relação importante e inseparável entre domínios matemáticos, cognitivos e afetivos.

O objetivo principal deste estudo é avaliar o impacto das variáveis cognitivas, emocionais e atitudinais no desempenho a matemática em alunos do $4^{\circ}$ ano de escolaridade, usando os dados recolhidos no âmbito do projeto Promoção de Mudanças na Aprendizagem Comunidades Escolares de Aprendizagem Gulbenkian XXI.

\section{Método}

O objetivo principal deste estudo é avaliar o impacto das variáveis cognitivas, emocionais e atitudinais, no desempenho a matemática em alunos do $4^{\circ}$ ano de escolaridade em função das notas académicas no final do ano letivo, provenientes de 6 agrupamentos de escolas portuguesas.

\section{Participantes}

Participaram no estudo 150 rapazes (52,3\%) e 137 raparigas $(51,8 \%)$, com idades compreendidas entre os 8 e 13 anos de idade $(\mu=9,66 \pm 0,77)$.

\section{Instrumentos}

Os dados foram recolhidos recorrendo a um conjunto de instrumentos disponíveis para a população portuguesa. Para avaliar variáveis atitudinais aplicou-se o Questionário de Atitudes face à Matemática (QAFM, Silva \& Candeias, 2012), que avalia quatro dimensões da atitude: sentimentos positivos, sentimentos negativos, competência percebida e crenças. Para avaliar o desempenho cognitivo e raciocínio aplicaram-se as Matrizes Progressivas Coloridas de Raven (MPCR, Simões, 1996) e a Bateria de Provas de Raciocínio (BPR, Almeida \& Lemos, 2006) que avalia quatro dimensões: raciocínio verbal, numérico, resolução de problemas e raciocínio abstrato. Por último, para avaliar as variáveis emocionais, recorreu-se ao Questionário de Inteligência Emocional Traço (TEIQ) (Petrides, 2012) o qual avalia a inteligência emocional em função de 4 dimensões: autocontrolo, bem-estar, emocionalidade e sociabilidade.

\section{Procedimento}

No âmbito do Promoção de Mudanças na Aprendizagem - Comunidades Escolares de Aprendizagem Gulbenkian XXI, aplicou-se um conjunto de provas de índole cognitiva, emocional e atitudinal. Os dados foram inseridos e processados no software SPSS Statistics 22. Todos os instrumentos de recolha de dados foram aplicados coletivamente em contexto da sala de aula, durante o horário letivo, na presença dos investigadores e dos professores das turmas. Os dados sobre o rendimento dos alunos participantes foram recolhidos junto das escolas no final do ano letivo. A participação foi voluntária e anónima. O consentimento informado dos pais por escrito, a autorização dos Diretores das Escolas e as autorizações do Comité Nacional de Proteção de Dados e da Direção Geral da Educação do Ministério da Educação por intermédio do sistema MIME - Monitorização de Investigação em Meio Escolar foram obtidas antes da recolha de dados. Todos os dados permaneceram confidenciais e anónimos.

\section{Resultados}

Começámos por uma análise univariada da relação entre as notas a matemática e as dimensões das variáveis cognitivas, emocionais e atitudinais. Os valores omissos no QAFM foram substituídos pelo valor da mediana obtida para o conjunto da amostra. Após esta análise às respostas dos participantes foram realizados dois tipos de análises: Análise de Regressão Linear usando o método stepwise e os coeficientes de regressão estandardizados $(\beta)$, através do software SPSS Statistics 22, para avaliar a influência das variáveis cognitivas, emocionais e atitudinais nas classificações finais dos alunos na disciplina de Matemática no final do $4^{\circ}$ ano.

\section{Impacto das variáveis cognitivas, emocionais e atitudinais no rendimento a matemática}

Primeiramente, foram verificadas as correlações entre os itens para estabelecer a relação entre o rendimento a matemática e as variáveis cognitivas, emocionais e atitudinais (tabela 1). 
Tabela 1.

Correlações entre as variáveis cognitivas, emocionais, atitudinais e nota a matemática

\begin{tabular}{cc}
\hline Dimensões das Variáveis & Nota a Matemática \\
\hline Sentimento negativo & $-.202^{*}$ \\
Competência percebida & $.378^{* *}$ \\
Autocontrolo & $-.218^{* *}$ \\
Raciocinio abstracto & $-.462^{* *}$ \\
Raciocinio verbal & $-.423^{* *}$ \\
Resolução de problemas & $-.555^{* *}$ \\
Raciocinio numérico & $-.548^{* *}$ \\
Matrizes Coloridas & $-.380^{* *}$ \\
\hline
\end{tabular}

Identificou-se um modelo (tabela 2) que explica 53\% da variância, que conjuga cinco variáveis com poder preditivo para o desempenho a matemática neste grupo de alunos: resolução de problemas $(\beta=-.437 ; p=.000)$, raciocínio numérico $(\beta=-.258 ; \mathrm{p}=.002)$, emocionalidade $(\beta=-.224 ; \mathrm{p}=.003)$, competência percebida $(\beta=.410 ; \mathrm{p}=.000)$ e sentimento positivo $(\beta=$ $-.295 ; \mathrm{p}=.011)$.

Tabela 2.

Impacto das variáveis cognitivas, emocionais, atitudinais na nota a matemática

\begin{tabular}{|c|c|c|c|}
\hline \multicolumn{2}{|c|}{ V. preditivas } & Beta & $p$ \\
\hline \multicolumn{2}{|c|}{ Resolução de problemas } & -.437 & .000 \\
\hline \multicolumn{2}{|c|}{ Raciocínio numérico } & -.258 & .002 \\
\hline \multicolumn{2}{|c|}{ Emocionalidade } & -.224 & .003 \\
\hline \multicolumn{2}{|c|}{ Competência percebida } & .410 & .000 \\
\hline \multicolumn{2}{|c|}{ Sentimento positivo } & .295 & .011 \\
\hline $\mathrm{R}^{2}$ Ajust & $\mathrm{F}$ & & \\
\hline .53 & 23.1 & & \\
\hline
\end{tabular}

\section{Discussão/Conclusão}

A partir dos resultados encontrados neste estudo emerge uma conclusão fundamental. O rendimento escolar na disciplina de Matemática é influenciado por múltiplas variáveis de natureza psicológica: cognitivas (resolução de problemas e raciocínio numérico), emocionais e atitudinais (competência percebida e sentimento positivo). Como refere Oliveira e Kottel (2016), o processo ensino-aprendizagem, para além das variantes cognitivas, é mediado por uma série de atributos ligados à emoção e afetividade, tal como é observado neste grupo de alunos, no qual aparecem em primeiro lugar variáveis cognitivas como preditivas do rendimento a matemática, mas seguidamente é possível verificar o impacto das emoções.

Neste sentido, se atendermos às teorias e modelos sobre atitudes, mudança de atitudes e relação entre atitudes e comportamento, somos levados a pensar que a definição de políticas educativas dirigidas à melhoria do rendimento escolar em matemática deverá prever, de forma articulada um trabalho de reforço e incentivo de experiências positivas na disciplina de matemática, tal como tem vindo a ser defendido por autores de referência na última década (e.g., Gonzáles-Pienda et al., 2006 a, b; Ponte et al., 2007). É fundamental que se promova uma relação positiva com a disciplina curricular e a confiança dos alunos nas suas capacidades pessoais para trabalhar os seus conteúdos e métodos, ou seja, é crucial que se promova a autoconfiança nos conhecimentos e capacidades a matemática; a segurança para lidar com situações que envolvam a matemática na vida escolar e extraescolar; o interesse pela matemática enquanto elemento da cultura humana; e, ainda, a capacidade para reconhecer e valorizar o papel da matemática.

Em síntese, podemos finalizar salientando como é crucial olhar para o rendimento a matemática no $4^{\circ}$ ano de escolaridade através de uma abordagem multivariada, remetendo para a necessidade de transpor estes resultados para a intervenção psicopedagógica promotora do sucesso a matemática que incida sobre componentes cognitivas, emocionais e motivacionais.

\section{Referências}

Almeida, L. S. \& Lemos, G. C. (2006). Bateria de Provas de Raciocínio: Versões 5/6, 7/9 e 10/12 (Manual Técnico). Braga: Universidade do Minho.

Colomeischi, A. And Colomeischi, T. (2015). The Students 'Emotional Life and Their Attitude toward Mathematics Learning. Procedia - Social and Behavioral Sciences. 180. 744-750.

Efklides, A. (2009). The role of metacognitive experiences in the learning process. Psicothema, 21(1), 76-82.

Elias , M.J., Zins, J. E., Weissberg, R. P. , Frey,K.S., Greenberg, M.T., Haynes, N.M., Kessler, R., SchawabStone, M. E., \&Shriver, T.P. (1997). Promoting Social and emotional learning: Guidelines for educators. Alexandria, VA: Association for Supervision and Curriculum Development.

González-Pienda, J. A., Nuñez, J. C., Solano, P., Silva, E. H., Rosário, P., Mourão, R. et al. (2006a). Olhares de género face à matemática: uma investigação no ensino obrigatório espanhol. Estudos de Psicologia, 11(2), 135-141.

González-Pienda, J. A., Núñez, J. C., Alvarez, L., González, P., González-Pumariega, S., Roces, C. Castejóm, L., Solano, P., Bernardo, A., García, D., Silva, E. H., Rosário, P. \& Feio, L. S. R. (2006b). Las Actitudes Hacia las Matemáticas en Funcion del Contexto Educativo. Actas do VIII Congresso Galaico Português de Psicopedagogia (2. ${ }^{\mathrm{a}}$ ed.). Braga: Universidade do Minho.

Larkin, Kevin, and Robyn Jorgensen. 2016. "'I Hate Maths: Why Do We Need to Do Maths?' Using iPad Video Diaries to Investigate Attitudes and Emotions Towards Mathematics in Year 3 and Year 6 Students." International Journal of Science and Mathematics Education 14 (5):925-944. doi: 10.1007/s10763-0159621-x.

Leandro, R. N. (2006). Insucesso escolar na matemática: um (outro) olhar: percepção dos alunos do $6 .^{\circ}$ ano do 
Ensino Básico sobre o insucesso. Dissertação de Mestrado apresentada à Universidade do Minho, Braga.

McLeod, D.B. (1992). Research on affect in mathematics education: A reconceptualization. In D. A. Grouws (Ed.), Handbook of research on mathematics teaching and learning (pp. 575-596). New York: Macmillan.

Mohsenpour, M., Hejazi, E., Kiamanesh, A. (2008). The role of self-efficacy, achievement goals, learning strategies and persistence in math achievement of 11th Grade High Schools Students in Tehran. Journal of Educational Innovations, 24, 153-172.

Oliveira, D. \& Kotell, A. (2016). Determinantes comportamentais e emocionais do processo ensinoaprendizagem. Intersaberes. 5 (6), 1-12

Petrides, K. V. (2009). Psychometric properties of the Trait Emotional Intelligence Questionnaire. In C. Stough, D. H. Saklofske, and J. D. Parker, Advances in the assessment of emotional intelligence. New York: Springer. doi: 10.1007/978-0-387-88370-0_5

Ponte, J. P., Serrazinha, L., Guimarães, H. M., Breda, A., Guimarães, F., Sousa, H., Menezes, L., Martins, M. E. G. \& Oliveira, P. A. (2007). Programa de Matemática do Ensino Básico. Disponível em http://www.dgidc.minedu.pt/ensinobasico/index.php?s=directorio\&pid=7.1.

Rosário, P. (1997). Facilitar a aprendizagem através do ensinar a pensar. Revista de Psicopedagogia, Educação e Cultura, 1(2), 237-249.

Roesken, B., Hannula, M. S. \& Pehkonen, E. (2011). Dimensions of students' views ofthemselves as learners of mathematics. ZDM.The International Journal on Mathematics Education. 43, 497-506.

Silva, S., \& Candeias, A. A. (2012). Questionário de Atitudes Face à Matemática (QAFM) Desenvolvimento, Construção e Estudo Psicométrico com Crianças e Jovens do $2 .^{\circ}$ e $3 .^{\circ}$ Ciclos do Ensino Básico Português. Comunicação apresentada no I Seminário RED - Rendimento escolar $e$ desenvolvimento. Évora. http://hdl.handle.net/10174/7972

Simões, M. M. R. (2000). Investigação no âmbito da aferição nacional das matrizes progressivas coloridas de Raven (M.P.C.R.). Lisboa: Fundação Calouste Gulbenkian/Fundação para a Ciência e Tecnologia/Ministério da Ciência e da Tecnologia

Yenilmez, K., Girginer, N., \& Uzun, A O. (2007). Mathematics anxiety and attitude level of students of the faculty of economics and business administrator; The Turkey model. International Mathematical Forum, 2, 2007, no. 41, 1997 - 202. 